\title{
Lymphoepithelial cysts of the pancreas: The use of endoscopic ultrasound-guided fine-needle aspiration in diagnosis
}

\author{
Zamil Karim MD ${ }^{1}$, Blair Walker MD FRCPC ${ }^{2}$, Eric C Lam MD FRCPC ${ }^{3}$
}

\begin{abstract}
Z Karim, B Walker, EC Lam. Lymphoepithelial cysts of the pancreas: The use of endoscopic ultrasound-guided fine-needle aspiration in diagnosis. Can J Gastroenterol 2010;24(6):348350.

Lymphoepithelial cysts (LECs) are rare non-neoplastic lesions that can appear as a complex cyst or a mass in the pancreas. Cytology from endoscopic ultrasound-guided fine-needle aspiration (EUS-FNA) can be helpful in making a diagnosis with the aim of avoiding unnecessary surgical resection. A case involving a 51-year-old woman with lower abdominal pain who was found to have a multiloculated cystic lesion at the junction of the pancreatic body and tail is described. Cytology from EUS-FNA was consistent with a pancreatic LEC. The lesion was managed conservatively and follow-up imaging of the cyst over the following two years was unchanged. The patient remains clinically well. Cytology from EUS-FNA can help distinguish LECs from cystic neoplasms, thus preventing radical surgical resection of this benign pancreatic cyst.
\end{abstract}

Key Words: Cytology; Endoscopic ultrasound; Lymphoepithelial cysts; Pancreas

\section{Les kystes lymphoépithéliaux du pancréas : Le recours à l'aspiration à l'aiguille sous échoguidage pour poser le diagnostic}

Les kystes lymphoépithéliaux (KLE) sont des lésions non néoplasiques rares qui peuvent prendre la forme d'un kyste complexe ou d'une masse dans le pancréas. La cytologie par aspiration à l'aiguille sous échoguidage (AAE) peut permettre de poser un diagnostic tout en évitant une résection chirurgicale inutile. Est décrit le cas d'une femme de 51 ans ayant des douleurs abdominales basses chez qui on a découvert des lésions kystiques multiloculées à la jonction du corps et de la queue du pancréas. La cytologie par AAE était compatible avec un KLE du pancréas. La lésion a été traitée de manière prudente, et l'imagerie de suivi du kyste au cours des deux années suivantes n'a pas évolué. La patiente est demeurée bien sur le plan clinique. La cytologie par AAE peut contribuer à distinguer un KLE d'un néoplasme, évitant ainsi la résection chirurgicale radicale de ce kyste bénin du pancréas.

\begin{abstract}
Pystic lesions of the pancreas can be divided into true cysts, pseudocysts and cystic neoplasms. A true cyst is distinguished by the presence of an epithelial lining, their benign natural history and developmental origin. Lymphoepithelial cysts (LECs) are a rare type of true cyst that can mimic pseudocysts and cystic neoplasms. Therefore, it is imperative to find a reliable way to diagnose LECs to avoid unnecessary radical surgical resection. The present report describes a 51-year-old woman in whom the diagnosis of an LEC was made on cytology by endoscopic ultrasound-guided fine-needle aspiration (EUS-FNA), who was thus managed conservatively.
\end{abstract}

\section{CASE PRESENTATION}

A 51-year-old woman presented with a one-month history of left lower quadrant abdominal pain. Her medical history was significant for sarcoidosis and type 1 diabetes. There was no history of alcohol use, gallstone disease or acute pancreatitis. An abdominal computed tomography (CT) scan revealed a multiloculated cystic lesion measuring $4.2 \mathrm{~cm} \times 2.6 \mathrm{~cm} \times 3.2 \mathrm{~cm}$ in the proximal body of the pancreas, with some minor peripancreatic lymphadenopathy. She had not undergone previous abdominal imaging. A follow-up CT scan one month later showed no change in the size of the lesion, but resolution of the lymphadenopathy was evident. Serum carbohydrate antigen
(CA) 19-9 was elevated at $350 \mathrm{U} / \mathrm{L}$ (reference range lower than $37 \mathrm{U} / \mathrm{L}$ ); the remainder of her laboratory results, including a complete blood count, electrolytes and liver enzymes, were normal.

Endoscopic ultrasound was performed, revealing a solid mass at the pancreatic neck measuring $2.5 \mathrm{~cm}$ in diameter (Figure 1). There was no accompanying ductal dilation. Several enlarged celiac and peripancreatic lymph nodes were seen. An EUS-FNA of the largest celiac lymph node showed benign lymphocytes, but the aspirate from the pancreatic mass revealed large amounts of well-differentiated squamous epithelium, keratinaceous and amorphous debris, and the presence of lymphoid cells (Figure 2). There were no suspicious features of carcinoma. A subsequent magnetic resonance (MR) image showed a well-defined, multilocular cystic lesion at the junction of the body and tail, measuring $2.4 \mathrm{~cm} \times 2 \mathrm{~cm}$ (Figure 3). On fat-suppressed T1 sequence, the cyst showed a high heterogeneous signal and an intermediate signal on T2 MR imaging, with no solid component.

A diagnosis of LEC was made based on the fine-needle aspirate of the pancreatic mass and the MR image. The patient was followed with a repeat MR imaging study six months later that showed no interval change. She continued to undergo abdominal ultrasound every six months for the next two years, with the lesion remaining unchanged. Currently, the patient continues to do well clinically.

${ }^{1}$ Division of General Internal Medicine; ${ }^{2}$ Department of Pathology and Laboratory Medicine; ${ }^{3}$ Division of Gastroenterology, Department of Medicine, University of British Columbia, Vancouver, British Columbia

Correspondence: Dr Eric C Lam, Division of Gastroenterology, Department of Medicine, University of British Columbia,

770-1190 Hornby Street, Vancouver, British Columbia V6Z 2K5. Telephone 604-688-6332 ext 224, fax 604-689-2004, e-mail ecslam@shaw.ca

Received for publication September 24, 2009. Accepted November 3, 2009 


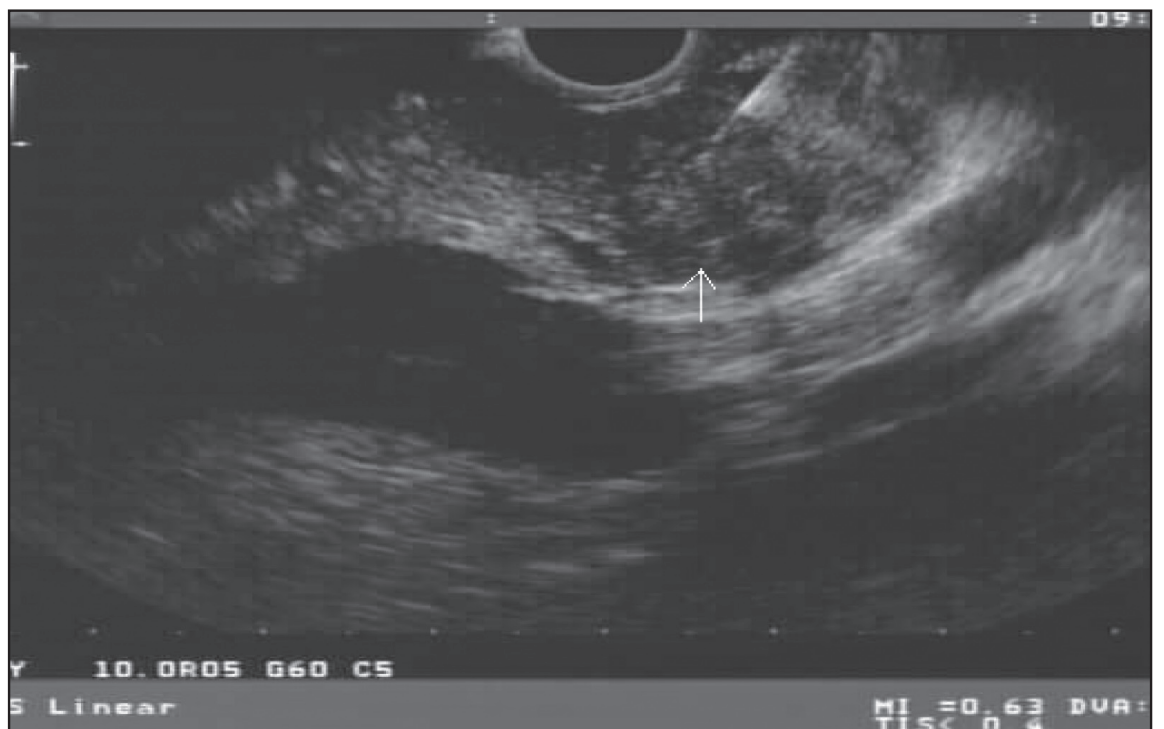

Figure 1) A $10 \mathrm{MHz}$ linear endoscopic ultrasound view of a lymphoepithelial cyst in the body of the pancreas during a fine-needle aspiration biopsy

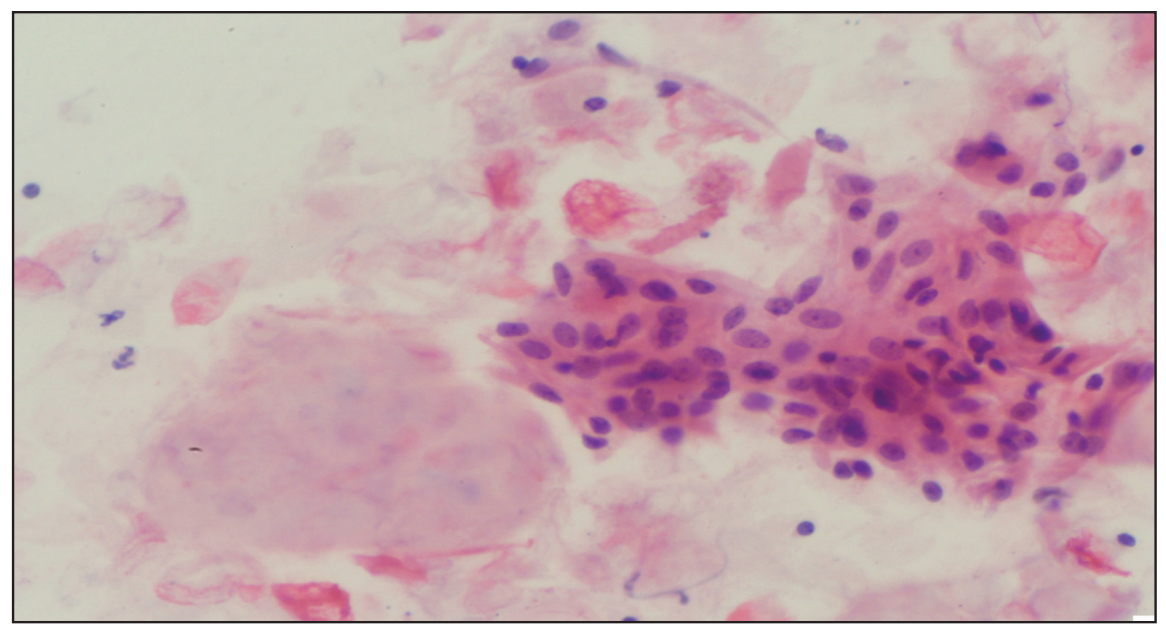

Figure 2) High-power view of cytology from the pancreatic cyst. Fine-needle aspirate showing well-differentiated squamous epithelium, keratinaceous and amorphous debris, and some lymphoid cells

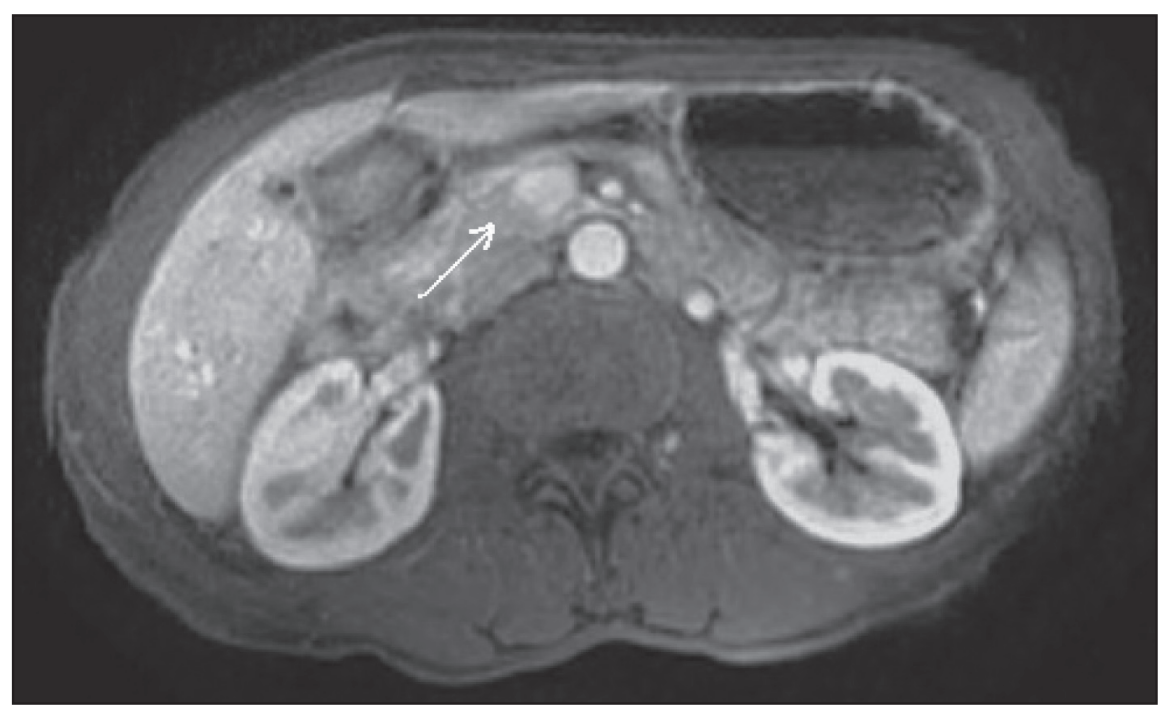

Figure 3) Fat-suppressed T1-weighted magnetic resonance image showing a well-defined cystic lesion at the junction of the pancreatic body and tail (arrow), emitting a high heterogeneous signal 


\section{DISCUSSION}

LECs are rare, nonmalignant lesions that were first described by Lüchtrath and Schriefers in (1) 1985. Since then, fewer than 90 cases have been reported in the English literature. The etiology of LECs remains unclear. Several hypotheses have been proposed, including that these lesions represent squamous metaplasia of either an obstructed intrapancreatic duct or a peripancreatic lymph node, or that they originate from branchial cleft cysts fused with the pancreatic anlage during embryogenesis $(2,3)$. LECs have been described in the parotid and submandibular glands, lung, thyroid and cervical regions (3). To date, there have been no reports of LECs becoming malignant or recurring after surgical resection. The cysts can occur anywhere in the pancreas, most commonly in the tail and body, followed by the head and neck, and have been described as being up to $13 \mathrm{~cm}$ in size $(3,4)$.

Clinically, LECs are usually found incidentally, most commonly in middle-aged men during investigations for abdominal symptoms when symptomatic patients can present with abdominal pain, nausea, vomiting, anorexia and weight loss. Physical examination is usually noncontributory. The differential diagnosis includes pseudocysts and cystic neoplasms.

The main issue in the diagnosis of LECs is differentiating them from other cystic lesions of the pancreas, particularly mucinous cystic neoplasms, because the natural history and management of these lesions are very different. The use of imaging, tumour markers, cytology and histology can be helpful in making a diagnosis. CT findings are often nonspecific and may demonstrate a low-attenuated unilocular or multilocular cystic lesion with a thin enhancing rim, as in the present case. However, LECs can also appear as a low-attenuated solid mass due to the large amount of debris and keratinous material often contained within the cyst $(5,6)$. Transabdominal ultrasound and EUS can be used to further support the cystic nature of these lesions. In an EUS case series by Nasr et al (6), pancreatic LECs were commonly found to appear as solid, hyperechoic, heterogenous masses with subtle postacoustic enhancement. As such, it has characteristics of both a mass lesion and a cyst. This was also the case in our patient. Fatsuppressed T1-weighted MR imaging can be more helpful because the high keratin content of LECs would show a high signal in T1- and low signal in T2-weighted imaging. However,

\section{REFERENCES}

1. Lüchtrath H, Schriefers KH. A pancreatic cyst with features of a so-called branchiogenic cyst. Pathologe 1985;6:217-9.

2. Truong LD, Rangdaeng S, Jordan PH. Lymphoepithelial cyst of the pancreas. Am J Surg Pathol 1987;11:899-903.

3. Truong LD, Stewart MG, Hao H, et al. A comprehensive characterization of lymphoepithelial cyst associated with the pancreas. Am J Surg 1995;170:27-32.

4. Mandavilli SR, Port J, Ali SZ. Lymphoepithelial cyst of the pancreas: Cytomorphology and differential diagnosis on fine needle aspiration. Diagn Cytopathol 1999;20:371-4.

5. Langan R, Paragi P, Guarino R, et al. Lymphoepithelial cysts of the pancreas: A sheep in wolves clothing. Am Surg 2009;75:520-2.

6. Nasr J, Sanders M, Fasanella K, et al. Lymphoepithelial cysts of the pancreas: An EUS case series. Gastrointest Endosc 2008;68:170-3. in our patient, the lesion showed moderate to high signal in both T1- and T2-weighted images.

Tumour markers are less specific. Serum CA 19-9 has been established as a sensitive marker of biliary and exocrine pancreatic cancer; however, despite several case reports $(6,7)$ of LECs with high serum CA 19-9 levels, including the one presented here, there seems to be no clear correlation between the two. Similarly, there have been conflicting reports (5,7-9) on the utility of cyst fluid analysis for CA 19-9, CA 125, amylase and carcinoembryonic antigen.

Cytology from EUS-FNA is a well-established modality for diagnosing most pancreatic cystic lesions. For many purely cystic lesions, cytology can be difficult because of acellular aspirates. However, for LECs, at least 20 cases have been reported in which the diagnosis was made based on CT or EUS-FNA (4,6,8-10). Cytology classically shows abundant anucleated squamous cells, multinucleated giant cells, mature lymphocytes in a background of keratinaceous debris and a lack of neoplastic cells $(4,8,10)$. Most of these features were visible on the cytology sample from our case (Figure 2); a diagnosis of a pancreatic LEC was made based on these results. Follow-up imaging has thus far confirmed the benign course of this lesion.

Interpretation of LECs by EUS-FNA is complicated by frequent contamination of the aspirate by tissues acquired by the needle during the procedure such as with mucinous and glandular epithelium from intestinal sources, making cystic neoplasm difficult to rule out. Very often, LECs can even contain a thick milky, creamy or frothy aspirate, further confusing the diagnosis (6). In the present case, squamous cells were seen but the presence of keratinaceous debris in the aspirate make contamination from the esophagus unlikely. Furthermore, as described by Renou et al (9), the absence of LEC cytology does not rule out a diagnosis of LEC. The difficulty in preoperative diagnosis still leads many LECs to surgical resection.

LECs of the pancreas are a rare, clinically benign lesion that should be considered in the differential diagnosis of any pancreatic cyst. A definitive preoperative diagnosis can prevent radical surgical resection, but can be difficult because LECs can often mimic neoplastic cysts of the pancreas (11). Our case illustrates the diagnostic utility of EUS-FNA, and supports the conclusion that if a patient is largely asymptomatic and cytology from EUSFNA strongly suggests the diagnosis of LEC, one can safely opt for conservative management with follow-up imaging.

7. Yamaguchi T, Takahashi H, Kagawar R, et al. Lymphoepithelial cyst of the pancreas associated with elevated CA 19-9 levels. J Hepato Pancreat Surg 2008;15:652-4.

8. Ahlawat S. Lymphoepithelial cyst of the pancreas: Role of ultrasound guided fine needle aspiration. JOP 2008;9:230-4.

9. Renou C, Giovannini M, Monges G, et al. Pitfalls of cyst fluid findings obtained by endoscopic ultrasonography fine-needle aspiration on a pancreatic lymphoepithelial cyst. Am J Gastroenterol 2007;102:213-5.

10. Zou XP, Li YM, Li ZS, et al. Lymphoepithelial cyst of the pancreas: A case report. Hepatobiliary Pancreat Dis Int 2004;3:155-7.

11. Anagnostopoulos PV, Pipinos II, Rose WW, et al. Lymphoepithelial cyst in the pancreas: A case report and review of the literature. Dig Surg 2000;17:309-14. 


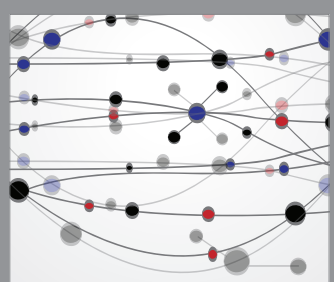

The Scientific World Journal
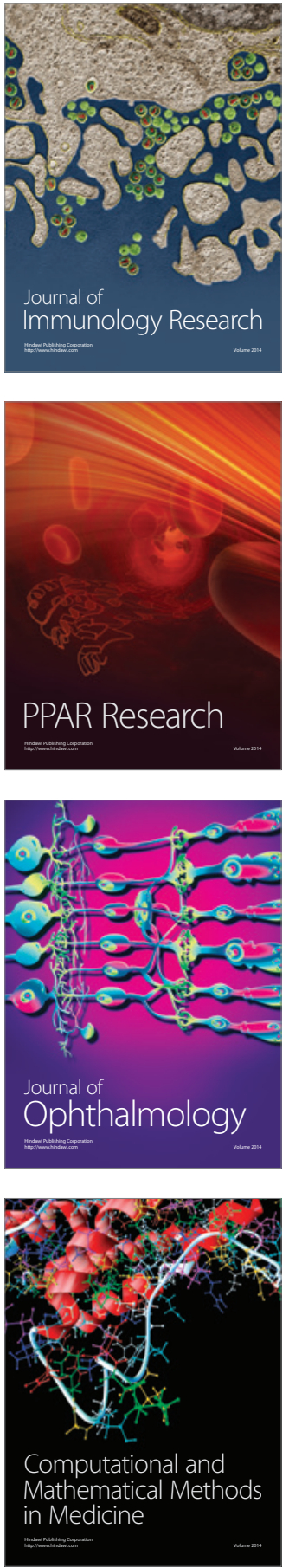

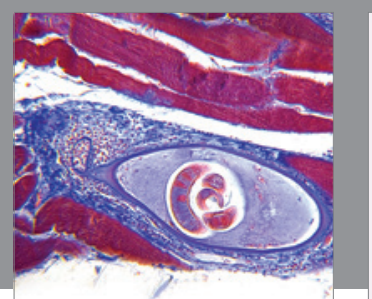

Gastroenterology Research and Practice

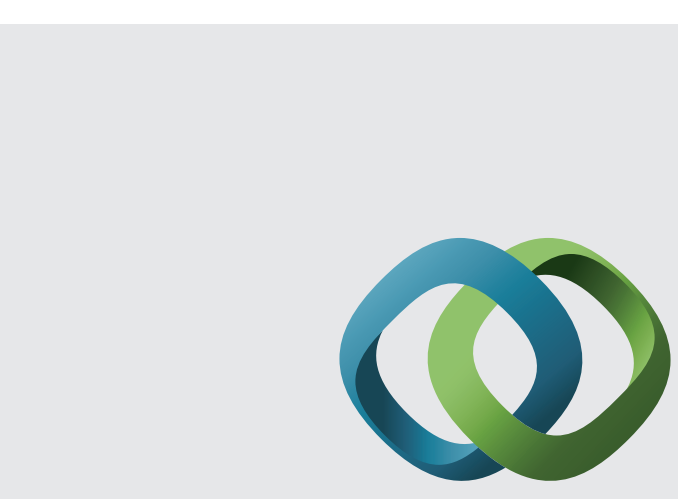

\section{Hindawi}

Submit your manuscripts at

http://www.hindawi.com
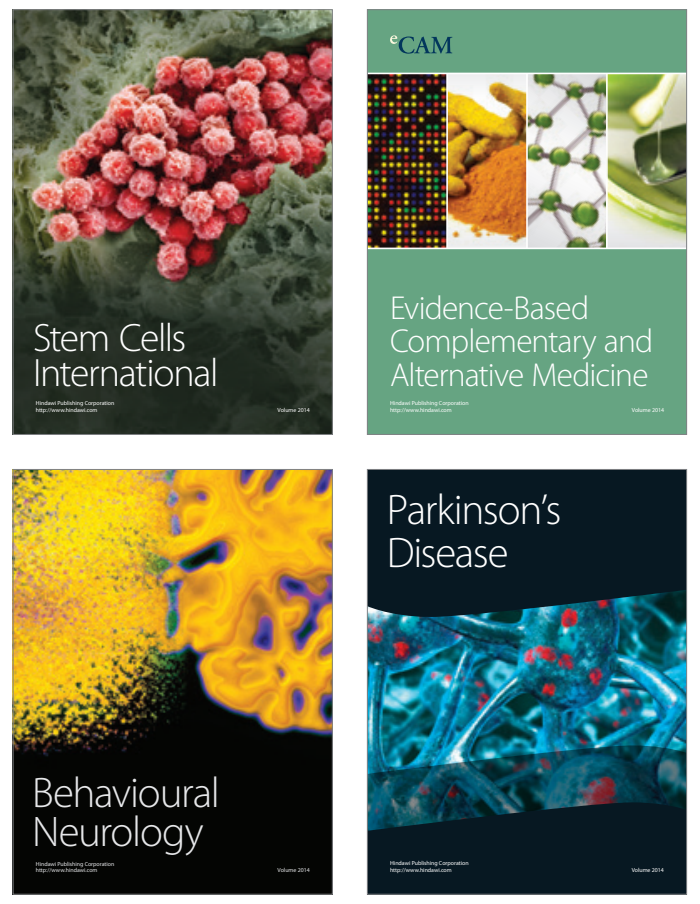
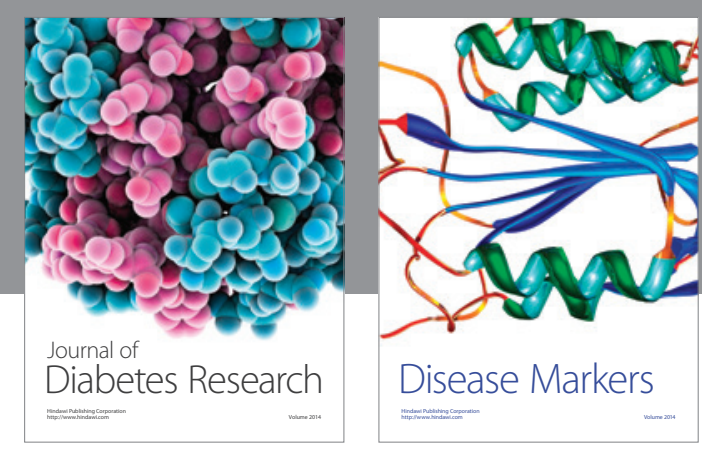

Disease Markers
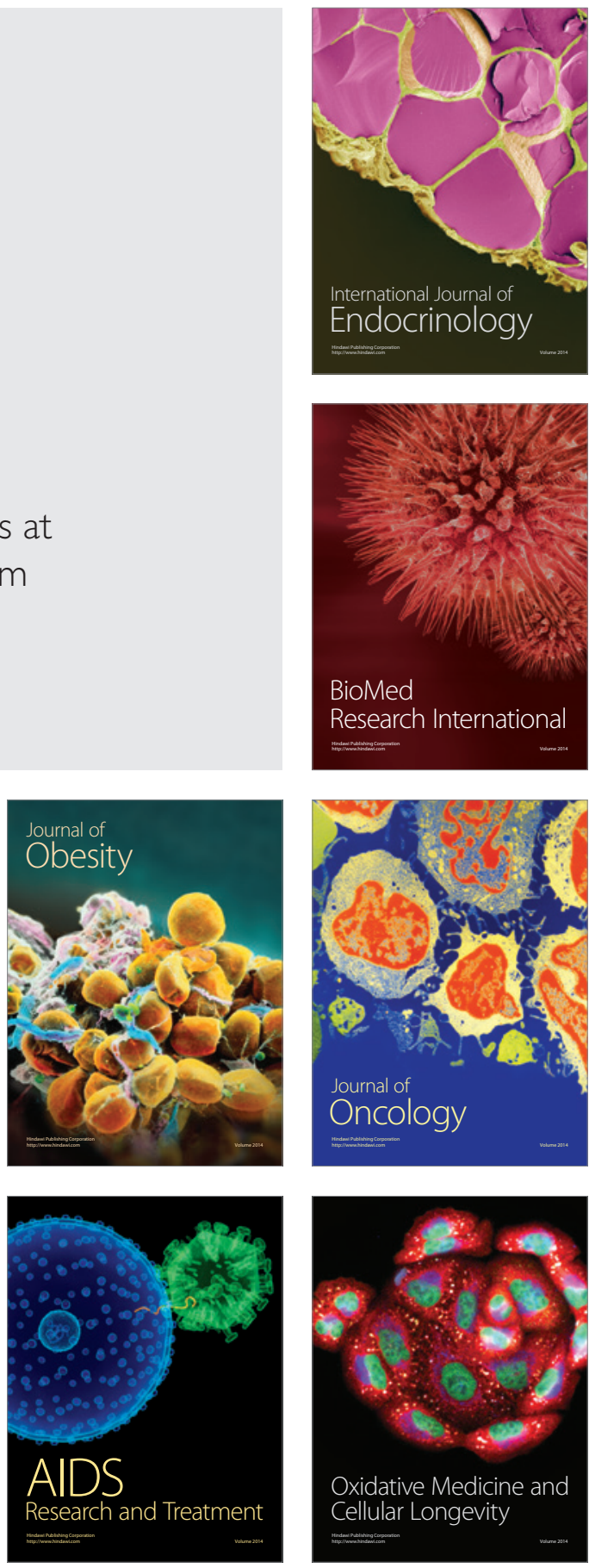